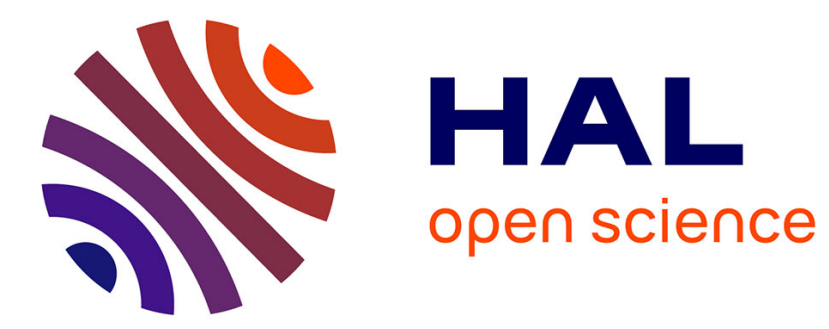

\title{
Mobilisation au travail par les compétences : l'apport de l'étude des groupes activistes
}

\author{
Fabien Hildwein
}

\section{To cite this version:}

Fabien Hildwein. Mobilisation au travail par les compétences : l'apport de l'étude des groupes activistes. RIMHE : Revue Interdisciplinaire Management, Homme(s) \& Entreprise, 2017, 26 (2), pp.49. 10.3917/rimhe.026.0049 . hal-02099439

\section{HAL Id: hal-02099439 \\ https://hal.science/hal-02099439}

Submitted on 15 Apr 2019

HAL is a multi-disciplinary open access archive for the deposit and dissemination of scientific research documents, whether they are published or not. The documents may come from teaching and research institutions in France or abroad, or from public or private research centers.
L'archive ouverte pluridisciplinaire HAL, est destinée au dépôt et à la diffusion de documents scientifiques de niveau recherche, publiés ou non, émanant des établissements d'enseignement et de recherche français ou étrangers, des laboratoires publics ou privés. 
Article de recherche

\section{MOBILISATION AU TRAVAIL PAR LES COMPÉTENCES : L'APPORT DE L'ÉTUDE DES GROUPES ACTIVISTES}

Fabien Hildwein

ARIMHE | « RIMHE : Revue Interdisciplinaire Management, Homme \& Entreprise » $2017 / 2 n^{\circ} 26$ | pages 49 à 72

ISSN 2259-2490

Article disponible en ligne à l'adresse :

http://www.cairn.info/revue-rimhe-2017-2-page-49.htm

\section{Pour citer cet article :}

Fabien Hildwein, « Mobilisation au travail par les compétences : l'apport de l'étude des groupes activistes », RIMHE : Revue Interdisciplinaire Management, Homme \& Entreprise 2017/2 ( $\left.\mathrm{n}^{\circ} 26\right)$, p. 49-72.

DOI 10.3917/rimhe.026.0049

Distribution électronique Cairn.info pour ARIMHE.

(C) ARIMHE. Tous droits réservés pour tous pays.

La reproduction ou représentation de cet article, notamment par photocopie, n'est autorisée que dans les limites des conditions générales d'utilisation du site ou, le cas échéant, des conditions générales de la licence souscrite par votre établissement. Toute autre reproduction ou représentation, en tout ou partie, sous quelque forme et de quelque manière que ce soit, est interdite sauf accord préalable et écrit de l'éditeur, en dehors des cas prévus par la législation en vigueur en France. Il est précisé que son stockage dans une base de données est également interdit. 


\section{Article de recherche \\ Mobilisation au travail par les compétences : l'apport de l'étude des groupes activistes}

Fabien HILDWEIN ${ }^{10}$

\section{Résumé}

Cet article explore la façon dont les compétences sont susceptibles de mobiliser les acteurs au sein d'une organisation. Pour ce faire, il considère les compétences non comme des ressources stratégiques au service de l'entreprise, mais comme des ressources facteurs de mobilisation au travail pour les individus. Le cadre théorique de la mobilisation des ressources, issu de la sociologie des mouvements sociaux, permet de saisir cette dimension des compétences. La partie empirique provient d'un travail ethnographique de 12 mois effectué auprès du groupe activiste féministe « La Barbe ». Elle explore comment une organisation disposant de peu de ressources peut mobiliser des individus en leur apportant des compétences cohérentes avec leurs parcours individuels et répondant à leurs attentes concernant notamment la recherche de l'émancipation personnelle. Les enseignements et les limites pour la mobilisation au travail par les compétences dans les organisations sont ensuite discutés pour conclure notamment sur les apports de l'étude pour la fidélisation des talents.

\section{Mots clés}

Compétences, mobilisation au travail, sociologie des mouvements sociaux, groupes activistes, démarche ethnographique.

\footnotetext{
${ }^{10}$ Docteur en Sciences de Gestion HEC Paris, ATER LARGEPA (EA 3386) - fabien.hildwein@ gmail.com Cet article a fait l'objet d'une communication en octobre 2016 à l'EM de Strasbourg lors du $27^{\text {ème }}$ congrès de l'AGRH « Retour vers le futur pour la GRH ».
}

RIMHE - Revue Interdisciplinaire Management, Homme \& Entreprise $\mathrm{n}^{\circ} 26$ - Printemps 2017 


\title{
La mobilisation au travail par les compétences : l'apport de l'étude \\ des groupes activistes - Fabien HILDWEIN
}

\begin{abstract}
This article explores the way in which skills may mobilize individuals within an organization. To that end, it considers skills rather as mobilizing resources for individuals than strategic resources for the firm. This suggests other ways through which skills can contribute to the firm and HR policies. The theoretical framework of resource mobilization, coming from the sociology of social movements, provides explanations in this regard. The empiric part is based upon a twelve-month ethnographic work among the feminist activist group "La Barbe". This part explores how an organization with few resources can mobilize and retain its members by providing skills which are coherent with their individual paths (especially the search for personal emancipation). Recommendations and the limits for human resources management are discussed to conclude, in particular, on the mobilization of talents.
\end{abstract}

\section{Keywords}

Skills, mobilization at work, social movements, activist group, ethnography. 


\section{La mobilisation au travail par les compétences : l'apport de l'étude \\ des groupes activistes - Fabien HILDWEIN}

\section{Introduction}

Les compétences sont considérées par les managers comme des ressources stratégiques au service de l'entreprise. Elles sont alors perçues comme des atouts individuels ou collectifs contribuant directement à la performance globale et représentent sous cet angle des instruments de gestion. Cependant, cette approche court le risque de ne prendre en compte qu'une seule dimension des compétences, alors qu'il s'agit d'un concept polysémique (Le Deist et Winterton, 2005) aux nombreux effets indirects sur la performance et la stratégie de l'entreprise (Chowhan, 2016).

Cet article se propose d'explorer un autre mode de contributions des compétences aux politiques de gestion des ressources humaines (GRH) et au projet d'entreprise, en les appréhendant en tant que facteur de mobilisation des collaborateurs (Tremblay et Wils, 2005 ; Tremblay et Simard, 2005, Tremblay et al., 2005). Notre objectif est de mettre en évidence comment l'acquisition de compétences spécifiques (par leur rareté, par le gain d'employabilité qu'elles permettent, par leur cohérence avec un parcours personnel, etc.) encourage les individus à s'attacher à une organisation et à s'investir dans un projet collectif. Nous nous inscrivons dans une démarche consistant à «passer d'une logique d'emploi à une GRH fondée sur la prise en compte du capital de compétences des individus qui forment le personnel $\gg$ (Retour, 2005, p. 187-188).

La question de recherche au cœur de ce travail porte donc sur la façon dont les compétences peuvent être facteurs de mobilisation au travail dans les organisations et sur les caractéristiques des compétences mobilisatrices pour les individus concernés.

Pour cela nous la sociologie des mouvements sociaux nous a fourni un cadre théorique fécond pour saisir le lien entre les deux phénomènes jusqu'ici dissociés, celui du développement des compétences et celui de la mobilisation au travail. Ce courant de recherche s'intéresse en effet à la façon dont des individus se mobilisent pour une cause (féminisme, antiracisme, écologie...) et, ce faisant, se forment pour y parvenir. Dans ce prolongement, la partie empirique explore ce lien entre compétences et mobilisation en étudiant comment des activistes du groupe féministe «La Barbe » expliquent leur propre investissement au sein de cette organisation. La discussion aborde ensuite les enseignements et les limites de l'étude. Nous concluons sur ses apports pour la mobilisation des talents.

\section{Définition des concepts et cadre théorique de l'étude}

Dans cette partie, nous présentons la définition du concept de compétences et de la conception de la mobilisation au travail par les compétences, puis nous explicitions le cadre théorique de l'étude, la sociologie des mouvements sociaux, afin d'en souligner la pertinence et apporter des éléments de réponse à notre problématique. 


\section{La mobilisation au travail par les compétences : l'apport de l'étude des groupes activistes - Fabien HILDWEIN}

\subsection{Le concept de compétence}

Définir ce que sont les compétences et comment elles peuvent contribuer à l'entreprise représentent une tâche centrale pour la GRH (Retour, 2005). Malgré de nombreux travaux sur les référentiels de compétences (Oiry et Sulzer, 2002 ; Labruffe, 2005), cela reste ardu. Aubret et ses coauteurs (2002) montrent ainsi que si des définitions existent, elles sont souvent le produit de compromis opératoires entre les différentes parties prenantes, notamment les entreprises, les employés, les formateurs et les chercheurs en sciences de gestion ou en psychologie du travail. La position hiérarchique (Green et James, 2003) comme la forme organisationnelle (Grugulis, 2003) influent sur ce que les individus associent au terme de «compétence». Si les approches du concept de compétence sont diverses, tant à cause des nombreuses disciplines qui les abordent que des cultures managériales par zones géographiques, elles tendent néanmoins à recouvrir une même réalité (Bouteiller et Gilbert, 2005; Gilbert, 2006).

Dans cette étude, nous nous sommes essentiellement appuyés sur la typologie proposée par Le Boterf (2002). Celle-ci a le mérite d'être relativement facile à manier et de trouver un équilibre entre une généralisation excessive qui la rendrait inopérante et une trop grande précision qui en ferait un outil trop complexe à manier. De plus, elle répond au besoin d'une typologie holiste et multidimensionnelle (Le Deist et Winterton, 2005), c'est-à-dire couvrant de nombreux aspects des compétences en incluant notamment à la fois les dimensions cognitives, opérationnelles et comportementales. Cette typologie est bâtie sur la distinction entre savoir et savoirfaire. Les savoirs correspondent aux cadres d'analyse orientant l'action (savoirs théoriques), à la connaissance qu'un individu a du contexte dans lequel il agit (savoirs d'environnement) ou à sa connaissance des modes et des moyens d'action (savoirs procéduraux). Les savoir-faire, quant à eux, correspondent à la capacité à réaliser une opération à partir de savoirs procéduraux (savoir-faire formalisés), aux connaissances tacites issues de l'expérience (savoir-faire empiriques), à la capacité à interagir aisément dans un groupe social (savoir-faire relationnels) ou à la capacité d'analyser et de résoudre des problèmes (savoir-faire cognitifs). Cette typologie est complétée par la notion de savoir-être, qui rend compte du fait que certaines compétences ne tiennent pas seulement d'un savoir, mais aussi d'un rapport à soi et au monde. Ils diffèrent des savoir-faire relationnels, dans la mesure où ils ne couvrent pas seulement les relations d'un individu aux autres, mais aussi de son rapport à soi (voire à son corps), par exemple dans la confiance qu'un individu peut avoir en lui-même lorsqu'il se tient et agit dans une circonstance donnée.

Ce faisant, il faut rappeler les avertissements répétés de Le Boterf (2002) contre cette division artificielle : "La compétence du professionnel c'est la faculté d'utiliser cet équipement de façon pertinente » (Le Boterf, 2002, p. 115), c'est-à-dire qu'un savoir n'est vraiment une compétence que lorsqu'il est utilisé à bon escient dans un contexte

RIMHE - Revue Interdisciplinaire Management, Homme \& Entreprise $\mathrm{n}^{\circ} 26$ - Printemps 2017 


\section{La mobilisation au travail par les compétences : l'apport de l'étude \\ des groupes activistes - Fabien HILDWEIN}

professionnel particulier. Elle est donc indissociable de l'individu qui la porte et la réalise. D'autre part, la compétence n'est pas une addition de ressources, mais une combinaison où chaque élément fait système et qu'il faut considérer comme un tout. Il est impossible de séparer les savoirs, savoir-faire et savoir-être, ils n'existent qu'en interaction les uns avec les autres. Cette typologie est donc avant tout un découpage analytique, permettant de mieux penser le concept de compétence, qu'il convient de manipuler avec précautions.

\subsection{La dimension mobilisatrice des compétences}

Comment ces compétences contribuent-elles au projet de l'entreprise ? Dans une approche de l'entreprise basée sur les ressources (resource based view of the firm) trouvant son fondement dans les travaux de Penrose (1963), Wenerfelt (1984) et Prahalad et Hamel (1990), les compétences sont considérées comme des ressources de capital humain qui participent à la performance de l'entreprise au même titre que d'autres types de ressources (capital physique ou capital organisationnel). Les compétences contribuent à la performance de l'entreprise d'une part en augmentant la performance de chaque individu dans les situations de travail qu'il rencontre, et d'autre part en augmentant la performance collective de l'organisation (Michaux, 2005 ; Dietrich et al., 2010). Dans cette optique, un des enjeux stratégiques de la GRH est d'être capable d'anticiper les besoins futurs de l'entreprise en termes de compétences et ainsi de toujours faire évoluer les compétences dont dispose l'entreprise pour répondre à sa vision stratégique (Le Boulaire et Retour, 2008). Il est également d'identifier les compétences les plus utiles et les plus rares afin de construire un avantage concurrentiel. En plus de la difficulté à mettre en pratique une politique RH stratégique fondée sur les compétences (Defélix et Retour, 2008), cette approche se heurte à la critique selon laquelle elle limiterait le DRH et la fonction RH à un rôle étroit, ne prenant en compte qu'une de ses dimensions, celle de partenaire stratégique, en lui faisant négliger d'autres comme celle d'administrateur, de champion des employés ou d'agent de changement (Ulrich, 1996).

Cette critique plaide pour la recherche d'autres modes de contributions au projet de l'entreprise par les compétences. En ce sens, il est nécessaire de s'interroger sur la destination des compétences : il s'agit de ressources, mais pour qui et pour faire quoi ? Ainsi, les compétences servent les individus directement. Ce sont des ressources pour leurs parcours professionnels ; les acquérir est un facteur important de motivation pour eux, au point d'encourager leur pleine mobilisation au service d'un projet d'entreprise. Plusieurs chercheurs ont abordé les compétences comme un facteur de mobilisation au travail dans les organisations (Tremblay et al., 2005). Le Boterf a souligné l'intérêt des individus pour le «portefeuille de compétences » qu'une entreprise peut proposer aux salariés d'acquérir comme incitation à l'embauche (Le Boterf, 2015, p. 48). Une de ses propositions pour repenser les compétences s'intitule d'ailleurs «Se positionner en 


\section{La mobilisation au travail par les compétences : l'apport de l'étude des groupes activistes - Fabien HILDWEIN}

offreur de compétences et non seulement en demandeur d'emploi » (Le Boterf, 2010, p.92). Pour Tremblay et Wils (2005, p. 38) la mobilisation dans les organisations se définit comme: «une masse critique d'employés qui accomplissent des actions (faisant partie ou non de leur contrat de travail, rémunérées ou non) bénéfiques au bien-être des autres, de leur organisation et à l'accomplissement d'une œuvre collective. ». Ce concept est à distinguer d'autres concepts proches. Par opposition à la motivation, qui peut s'y limiter, la mobilisation suppose des comportements qui dépassent les attributions du poste, (Tremblay et Wils, 2005). Elle se distingue également de l'implication et de l'engagement au sens où ces derniers sont avant tout des attitudes psychologiques qui prédisposent aux comportements de mobilisation, mais ne l'assurent pas (Bichon, 2005).

La façon dont les compétences permettent de mobiliser les individus reste incertaine. Pour mieux saisir la relation entre mobilisation et compétences, nous proposons de faire appel à un modèle issu d'une autre discipline : la sociologie des mouvements sociaux.

\subsection{Cadre théorique de la sociologie des mouvements sociaux}

Le concept central de ce courant est celui de «mouvement social », défini comme « une communauté agissant avec un certain degré d'organisation et de continuité, hors des cadres institutionnels ou organisationnels classiques, dans le but de remettre en cause ou de défendre une autorité existante, qu'elle soit institutionnelle ou culturelle, dans le groupe, l'organisation, la société, la culture ou l'ordre du monde dont elle fait partie ${ }^{11}$ (Snow et al., 2004, p. 11). Un mouvement social est composé à la fois d'individus qui s'en revendiquent et se définissent souvent comme des «militants », ainsi que des organisations dont le but principal est de soutenir une ou plusieurs causes du mouvement. Ces organisations sont appelées des «organisations de mouvement social »(OMS). Les OMS peuvent être de natures différentes selon les moyens qu'elles utilisent pour soutenir leurs causes. Elles peuvent par exemple produire de l'information, offrir des services à des populations en difficulté ou encore exercer une pression médiatique pour influencer des responsables politiques ou d'entreprise.

Le courant de la mobilisation des ressources fait partie des premiers courants de la sociologie des mouvements sociaux (Fillieule et Péchu, 1993). Il tente d'expliquer l'action collective face au paradoxe d'Olson (1965). Selon ce dernier, l'action collective est impossible si l'on considère des individus rationnels : les gains collectifs sont importants mais les gains individuels sont largement dépassés par les risques et les coûts individuels de passer à l'action. Le comportement le plus rationnel est alors

\footnotetext{
${ }^{11}$ Traduction par l'auteur de : "Collectivities acting with some degree of organization and continuity outside of institutional or organizational channels for the purpose of challenging or defending extant authority, whether it is institutionally or culturally based, in the group, organization, society, culture, or world order of which they are part".
}

RIMHE - Revue Interdisciplinaire Management, Homme \& Entreprise $\mathrm{n}^{\circ} 26$ - Printemps 2017 


\section{La mobilisation au travail par les compétences : l'apport de l'étude des groupes activistes - Fabien HILDWEIN}

celui de «passager clandestin » consistant à ne pas agir et à bénéficier ensuite des gains collectifs obtenus. Les auteurs de ce courant (Jenkins, 1985; McCarthy et Zald, 1973 ; McCarthy et Zald, 1977) développent l'idée que les OMS rendent possible l'action collective en accumulant les ressources nécessaires. Les acteurs s'engagent dans une OMS parce qu'elle abaisse les coûts de l'action en leur donnant les moyens d'agir. Les premières ressources considérées étaient matérielles et humaines, mais d'autres types de ressources ont été identifiés, tels que le statut de l'OMS, l'identité qu'elle donne à ses membres, les compétences qu'elle leur permet d'acquérir, les réseaux avec lesquels elle les met en contact ainsi que les structures organisationnelles ou institutionnelles qu'elle propose (Pierru, 2010). Le courant de la mobilisation des ressources de la sociologie des mouvements sociaux permet d'expliquer la nécessité de développer des organisations au sein des mouvements sociaux, ainsi que le besoin qu'ont les individus de les rejoindre. Il permet de comprendre le rôle des organisations dans la mobilisation.

Plus spécifiquement McCarthy et Zald (1973, 1977) adoptent une approche dite entrepreneuriale des OMS dans la mesure où ils considèrent qu'elles sont en concurrence entre elles pour traiter les différentes causes portées par un mouvement social. Ils utilisent le terme d' «industrie d'un mouvement social». Plus une OMS défend efficacement une cause, plus elle gagne en légitimité et est susceptible d'accumuler des ressources. On peut d'autant plus légitimement qualifier ce modèle d'entrepreneurial que les auteurs reconnaissent explicitement que ce concept d'industrie de mouvement social fait écho à celui d'《 industrie» au sens économique du terme : «La définition d'une SMI (industrie d'un mouvement social) est à mettre en parallèle avec le secteur industriel en économie $»^{12}$ (McCarthy et Zald, 1977, p.1279).

Le courant de la mobilisation des ressources nous semble pouvoir contribuer à une meilleure compréhension du phénomène de mobilisation au travail par les compétences. D'une part, les OMS en concurrence pour mobiliser les individus pour leur cause peuvent être comparées aux entreprises cherchant à attirer les meilleurs candidats et à acquérir une position forte à long-terme sur le marché de l'emploi. D'autre part, la définition de la mobilisation se rapproche de la conception de la sociologie des mouvements sociaux. L'idée que la mobilisation est un phénomène collectif, qui concerne l'individu mais le dépasse et qui exige une masse critique d'individus pour advenir, rejoint tout à fait la préoccupation pour l'action collective de la sociologie des mouvements sociaux (Fillieule et Péchu, 1993). De même considérer que les individus mobilisés «accomplissent des actions bénéfiques au bien-être des

\footnotetext{
${ }^{12}$ Traduction par l'auteur de "The definition of SMI (social movement industry) parallels the concept of industry in economics".
}

RIMHE - Revue Interdisciplinaire Management, Homme \& Entreprise $\mathrm{n}^{\circ} 26$ - Printemps 2017 


\section{La mobilisation au travail par les compétences : l'apport de l'étude des groupes activistes - Fabien HILDWEIN}

autres, de leur organisation et à l'accomplissement d'une œuvre collective » (Tremblay et Wils, 2005, p. 38) correspond bien à la mobilisation au sein des OMS.

$\mathrm{Si}$ cette définition réunit dans une certaine mesure sciences de gestion et sociologie des mouvements sociaux, l'approche de ces deux disciplines a jusqu'ici été différente. De manière générale, la sociologie des mouvements sociaux tend soit à considérer la mobilisation avec une très grande ampleur dans le temps (Koopmans, 2004; Tarrow, 1983) et dans l'espace (Sommier, 2010; Tarrow, 2005; Tarrow et della Porta, 2005), soit à privilégier une approche psychologique par l'offre d'engagement (Klandermans, 2004) ou par les cadres d'analyse (Snow et Benford, 1988; Snow et al., 1986). Les sciences de gestion, au contraire, ont recherché les facteurs de mobilisation internes aux organisations, qu'il s'agisse d'un climat positif fondé sur la reconnaissance des efforts des individus (Tremblay et Simard, 2005) ou de pratiques de management plus concrètes (Tremblay et al., 2005). De plus, les effets de la mobilisation sur la rentabilité financière des entreprises sont restés l'une des préoccupations majeures des gestionnaires (Aït Razouk et Bayad, 2011; Barraud-Didier et al., 2003), ce qui n'est pas partagée par la sociologie des mouvements sociaux.

La question de recherche à l'origine de notre étude est celle des apports potentiels de l'étude d'un mouvement social par le courant de la mobilisation des ressources de la sociologie à la compréhension du phénomène de mobilisation au travail par les compétences dans les organisations.

\section{Méthodologie de l'étude empirique}

Dans l'objectif de mettre en exergue les compétences en tant que ressources mobilisatrices des individus, nous nous sommes focalisés sur l'étude des groupes activistes. Ces organisations représentent une catégorie particulière d'OMS qui se concentre sur les opérations visant à attirer l'attention sur des causes, dans le but d'en défendre l'importance et de leur donner de la légitimité. Il s'agit souvent d'organisations de petite taille, relativement peu structurées et disposant de ressources limitées. Leurs membres sont appelés des activistes. Pour être vus et entendus, les groupes activistes font appel à des «performances » qui permettent d'exprimer visuellement leur message et leur soutien à leur cause. La manifestation représente la performance la plus connue, mais il en existe bien d'autres (piquet, pétitions, actions sur les réseaux sociaux, happenings, ...). Ce terme est emprunté au monde du théâtre et renvoie aux pièces («performances » en anglais) que connaît une troupe et qu'elle utilise à chaque représentation. Ils ne disposent pas de ressources financières et s'appuient sur des engagements bénévoles. Les facteurs de mobilisation sont donc d'un autre ordre que la rémunération pour des collectifs témoignant par ailleurs d'une très forte mobilisation de leurs membres. Les groupes activistes en ressortent comme particulièrement pertinents pour notre étude. 


\section{La mobilisation au travail par les compétences : l'apport de l'étude des groupes activistes - Fabien HILDWEIN}

\subsection{Le terrain de l'étude : La Barbe, groupe d'action féministe}

Nous avons opté pour l'étude d'un groupe d'activistes, La Barbe, qui vise à dénoncer l'absence de femmes aux postes de pouvoir, qu'il s'agisse d'un pouvoir concret (postes de direction, représentation parmi les candidats d'un parti ou parmi les élus) ou d'un pouvoir symbolique (représentation parmi les experts et les intervenants durant une conférence ou dans un cercle de réflexion). Le groupe est composé d'une trentaine de femmes activistes agissant régulièrement, ainsi que d'une centaine de membres moins fortement impliquées mais sympathisantes et participant occasionnellement aux performances. En termes d'âge, elles sont réparties entre 25 et 65 ans assez uniformément, même si les fondatrices sont plus âgées, ayant déjà eu une carrière militante et féministe importante. En termes professionnels, elles occupent en grande majorité des professions intellectuelles supérieures: consultantes indépendantes, plasticiennes, journalistes, cinéastes, photographes, chercheuses, professeures des écoles, mais travaillent rarement en entreprise. Les plus jeunes poursuivent des études supérieures. La Barbe est volontairement une organisation fonctionnant avec une très faible structure organisationnelle (refus de toute hiérarchie, bureaucratie presque inexistante, processus de décision rapides).

La performance principale de La Barbe se déroule toujours selon le même protocole. Les activistes identifient une organisation (politique, économique, culturelle, humanitaire ou encore religieuse) dont les positions de pouvoir sont occupées en grande majorité par des hommes. Dès que l'organisation apparaît lors d'un événement public (notamment des assemblées générales et des conférences de presse), les activistes se rendent à cet événement et se fondent dans la foule. Une fois l'événement commencé, les activistes mettent sur leurs visages des barbes postiches, et montent sur l'estrade à proximité des intervenants, ou se rassemblent devant la scène. Elles restent silencieuses, calmes, avec les bras croisés. Deux d'entre elles déploient une banderole. L'une des activistes prend alors la parole ou s'exprime au micro. Elle lit un texte préparé à l'avance, dans lequel les activistes félicitent ironiquement l'organisation d'avoir su garder les femmes hors des lieux de pouvoir. Puis les activistes serrent les mains des intervenants et quittent rapidement les lieux. Le texte du tract est distribué au public et les photos de l'action et le texte du tract sont diffusés à une liste de journalistes ainsi que sur les réseaux sociaux Facebook et Twitter.

En elle-même, la nature de la performance témoigne de la forte mobilisation des personnes pour affronter la situation et la mener à bien.

\subsection{Collecte et analyse des données}

Cette partie empirique s'appuie sur une enquête de type ethnographique de 12 mois entre juillet 2011 et juillet 2012 auprès du groupe menée par observation participante et des entretiens auprès des acteurs centraux, complétés par la collecte de tous les 


\section{La mobilisation au travail par les compétences : l'apport de l'étude des groupes activistes - Fabien HILDWEIN}

documents disponibles. Le travail ethnographique a aussi consisté en une immersion du chercheur dans la vie du groupe.

L'ensemble des données collectées se compose de 38 entretiens de membres de La Barbe, de journalistes ayant écrit sur La Barbe ou de responsables diversité des entreprises concernées par les actions de La Barbe, de notes d'observations couvrant 18 actions de La Barbe et 25 réunions internes, de 2325 photos des actions de La Barbe, de 226 articles de presse concernant La Barbe collectés sur Factiva et LexisNexis (voir annexe 2), de 93 communiqués de presse émis par La Barbe et de 138 tracts de La Barbe.

Les trois corpus de texte (entretiens, articles de presse et tracts) ont été analysés en les codant à l'aide d'un logiciel d'analyse de contenu (NVivo) qui a permis d'en tirer les thèmes principaux. Les données utilisées dans cet article proviennent des entretiens auprès de 18 activistes de La Barbe ainsi que des observations faites au cours des actions. Les entretiens font appel à la méthode du récit de vie (Bertaux, 1997; Sanséau, 2005) afin de capter le parcours militant et/ou féministe des activistes de La Barbe, pour comprendre par quelles étapes elles passent, quels choix elles effectuent, quelle est la part du hasard (rencontres fortuites, découvertes inattendues) et quel sens elles donnent a posteriori à cet engagement. A ces questions s'ajoute une deuxième série destinées à capter leur point de vue sur les performances réalisées ainsi que le sens qu'elles donnent aux différents éléments de ces performances. Un guide d'entretien a été utilisé (annexe 1). En accord avec les techniques d'entretien semi-directif pratiqués en ethnographie, les interviewés disposent de beaucoup de temps pour répondre et l'interviewer les encourage à poursuivre le fil de leurs pensées sans les influencer, dans le but de faire émerger des enjeux et des thèmes que le guide d'entretien ne couvrirait pas initialement. L'expérience et l'immersion au sein du groupe permettent à l'ethnographe de saisir pleinement ce qu'expriment les interviewés dans leurs entretiens. La démarche est interprétativiste. Elle vise à saisir les explications que les individus donnent à leur activité et, en la croisant avec nos observations, de décrire les événements et la subjectivité des individus qui les vivent et les réalisent (Beaud et Weber, 2010).

Le codage des entretiens des activistes a d'abord permis d'identifier les unités de sens, puis de les regrouper en catégories plus larges permettant de repérer les grands thèmes abordés par les activistes : Sens donné aux performances, Rapport au média, Facteurs de mobilisation et Fonctionnement interne du groupe. Le thème «Facteurs de mobilisation » se découpe dans les catégories suivantes : Affects (affects provoqués par l'action, gestion des affects par l'organisation), Compétences acquises (développé dans ce travail), Carrière militante (engagements dans d'autres mouvements sociaux, évolution personnelle et professionnelle) et Carrière féministe (évolution intime de la personne, à la fois sur le plan intellectuel et sur le plan de l'engagement activiste). Si les catégories «Carrière militante » et «Carrière féministes » étaient attendues, celles 


\section{La mobilisation au travail par les compétences : l'apport de l'étude des groupes activistes - Fabien HILDWEIN}

sur les «Affects » et les «Compétences acquises » ont émergé durant l'enquête, de même pour les thèmes sur le « Rapport au média » et le «Fonctionnement interne ». Bien que cette démarche soit proche de la grounded theory (Corbin et Strauss, 1990 ; Glaser et Strauss, 1967), nous avons pris en considération l'avertissement de ne pas regrouper aveuglément sous ce terme toutes les méthodologies qualitatives (Suddaby, 2009). En l'occurrence, la construction théorique de ce travail s'est faite par allersretours itératifs entre la théorie et les résultats du terrain, les concepts éclairant l'expérience ethnographique et celle-ci à son tour permettant de discuter les théories (Beaud et Weber, 2010), plutôt que de remonter du terrain vers les concepts comme dans la grounded theory.

Dans le cadre de cet article, la méthode ethnographique permet de comprendre l'expérience intérieure des activistes, notamment les émotions, l'évolution des cadres d'analyse et les compétences acquises au cours de l'action, qui relèvent toutes de la mobilisation des activistes. L'immersion du chercheur dans un contexte social particulier permet de faire pleinement ressortir le sens des actions, interactions et discours des acteurs (Rouleau et al., 2014) et, dans une perspective exploratoire, de capter des relations encore peu étudiées et difficiles à saisir, telles que celles entre acquisition de compétences et mobilisation. L'ethnographie semble d'autant plus indiquée pour aborder la notion de compétence que cette dernière ne peut être détachée de son contexte et de la façon dont leur propre activité professionnelle fait sens pour les acteurs (Capaldo et al., 2006). Ce travail s'inscrit ainsi dans le «tournant ethnographique » pris depuis une dizaine d'années par l'étude des organisations et du management (Cunliffe, 2010; Watson, 2011) qui aboutit aujourd'hui à un renouvèlement des méthodes ethnographiques (Rouleau et al., 2014).

\section{Résultats : le développement des compétences des activistes}

Les entretiens ont mis en évidence de manière inattendue au début de la recherche, l'importance des compétences dans la mobilisation des activistes. Plus précisément, deux éléments jouent un rôle : d'une part le contenu des compétences elles-mêmes, qui sont perçues comme émancipatrices par les activistes et correspondent donc aux raisons qui les ont poussées à entrer dans le groupe, et d'autre part les modes d'acquisition de ces compétences au sein du groupe activiste, qui sont intimement liés au contenu de ces compétences et qui sont inséparables de la participation au groupe.

\subsection{L'acquisition des compétences par les activistes}

Faire partie de La Barbe permet sur le long-terme d'acquérir deux types de compétences. Tout d'abord, participer à la vie et aux actions de La Barbe permet de développer une conscience militante et féministe, c'est-à-dire d'acquérir de nouveaux cadres d'analyse permettant d'interpréter la réalité dans chaque situation donnée (Goffman, 1974). Il s'agit de savoirs théoriques, puisqu'ils orientent l'action et lui 


\section{La mobilisation au travail par les compétences : l'apport de l'étude des groupes activistes - Fabien HILDWEIN}

donnent du sens. Les activistes décrivent ainsi leurs propres évolutions et leur adhésion graduelle au féminisme :

«Etre à La Barbe amène à se documenter pour les actions et à changer de lentille. C'est vraiment changer de regard à un moment. Je suis dans le bus, je suis à une réunion, je suis à un repas de famille, qu'est-ce qui se joue, combien on est d'hommes, combien on est de femmes, qui prend la parole, qui occupe l'espace, qui prend les décisions, quelque chose dont je n'avais absolument pas conscience, je pensais qu'on était égaux et voilà. Tu deviens un peu plus attentive à ce qui se passe dans les faits plutôt qu'à ce que tu ressens. Tu réalises que tous les aspects de la vie publique sont dominés par des hommes. C'est exactement ce sentiment-là : quelque part d'avoir changé de paires de lunettes». [Aline et Coraline - 21 septembre 2011]

Plus précisément, ce changement de regard représente l'adoption d'un cadre d'analyse féministe conscient, qui remplace des cadres antérieurs dont les individus avaient moins conscience. Ce dévoilement représente un tournant intérieur pour les activistes, pour qui des situations jusque-là vécues comme naturelles et allant de soi, sont subitement perçues comme problématiques, et imposent de passer à l'action.

Accompagnant cette évolution, les activistes mentionnent souvent leur découverte d'auteures et de cadres théoriques féministes, issus principalement du féminisme matérialiste (Delphy, 1979) et du féminisme queer (Butler, 1990). Mais le développement d'une conscience militante ne se limite pas à l'acquisition de nouveaux cadres ou d'éléments théoriques, il doit être compris comme une évolution intellectuelle au sens large :

Chercheur : "Tu m'as dit que tu étais dans les balbutiements du féminisme, qu'est-ce que ça signifie?»

Amelia : "Je savais pas comment militer, je savais pas quoi penser, ma pensée n'était pas construite, je sentais qu'il y avait un truc qui était dégueulasse mais je ne savais pas démêler les fils. On cherche, on sent qu'il y a un truc. On sent qu'il y a une connexion, même si ce n'est pas encore hyper limpide et hyper clair, c'est pour ça que je disais les balbutiements, l'histoire du féminisme j'y connaissais rien, pas plus que les grands noms du féminisme. » [Amelia - 20 mars 2013]

Le processus ne consiste pas uniquement en une accumulation de connaissances. Il s'agit d'un travail de clarification de la pensée, qui permet de passer d'un état où prédominent l'intuition et la recherche de cohérence, à une expression claire des idées et une adhésion explicite au féminisme. L'effort de " mise en ordre dans ses idées » permet d'affermir ses convictions. Il s'agit de savoir-faire cognitifs qui permettent de résoudre différentes questions à l'aide d'outils et d'approches neufs.

La conscience militante et féministe n'est pas tout et l'évolution des activistes dépasse le niveau intellectuel d'analyse et d'interprétation du monde. Elle concerne également leur rapport à l'espace public.

«L'occupation de l'espace, maintenant je fais beaucoup plus attention si je suis dans le métro, je suis une femme donc j'ai le droit d'être là aussi et donc je ne vais pas me recroqueviller comme j'avais tendance à le faire. Donc quand je marche, à me dire, et si y'a

RIMHE - Revue Interdisciplinaire Management, Homme \& Entreprise $\mathrm{n}^{\circ} 26$ - Printemps 2017 


\section{La mobilisation au travail par les compétences : l'apport de l'étude des groupes activistes - Fabien HILDWEIN}

un mec ou même une femme qui arrive devant, je ne vais pas me reculer, un peu ces réflexes que j'avais avant : "excusez-moi d'exister pardon, pardon ». Maintenant je sais que quand je m'installe sur un strapontin ou dans le métro, je m'installe, donc j'ai le droit, j'ai le droit de bousculer les autres si j'estime qu'ils prennent un peu beaucoup plus de place qu'ils ne devraient. » [Amelia - 20 mars 2013]

Il s'agit d'apprendre à revendiquer l'espace pour soi et de ne pas devoir justifier sa présence. Cette importance du corps (se sentir légitime à agir et à être présente dans un lieu de pouvoir), du rapport à soi (notamment dans la remise en cause de sa socialisation primaire) et du rapport aux autres (face à une autorité jugée illégitime) justifient d'utiliser le terme spécifique de «savoir-être » plutôt que de savoir-faire relationnels, qui ne concerneraient que le rapport aux autres, ou de savoir-faire empiriques, qui ne concerneraient que le rapport aux choses.

Le développement de la conscience politique et le développement de savoirs-être sont des processus profondément émancipateurs. Tout d'abord, la conscience féministe justifie de se penser comme sujet politique pleinement légitime :

«Devenir militante pour cette cause m'a ouvert le champ de la politique comme quelque chose dans lequel je pouvais intervenir. Alors qu'avant, en tant que fille, et là vraiment c'est de la construction de fille, j'osais même pas penser, je pensais même pas que je pouvais avoir une idée sur la politique, je laissais les autres penser pour moi, j’écoutais, je choisissais dans le panel de ce que les gens choisissaient comme idées, mais je n'osais pas me positionner moi, et je me positionnais par le choix des autres, comme sur un étalage. Et à un moment donné je me suis dit être féministe c'est faire de la politique. » [Anne-Louise - 2 février 2012]

A nouveau, l'émancipation n'est pas seulement un phénomène intellectuel, c'est aussi une autre manière de se considérer, en l'occurrence d'accepter son ambition et de se penser comme digne d'exercer son pouvoir au même titre que les hommes :

«La Barbe m'a apporté quelque chose dernièrement, c'est le fait d'assumer ses ambitions. Pour moi, l'ambition ça a toujours été connoté comme quelque chose de mal, mais en fait c'est connoté comme quelque chose de mal que pour les femmes, un mec ambitieux, ça le fait. Une femme ambitieuse : ah! beurk! Et du coup, je crois qu'il y a quelque chose de là aussi, quand on s'invite dans ces lieux de pouvoir, on va pas attendre que vous nous preniez par la main et que vous nous autorisiez à accéder à ces lieux de pouvoir, on fait irruption, et bon gré mal gré on est là. » [Justine - 20 janvier 2012]

Pour les activistes les plus anciennes, ce changement s'ancre profondément dans la personnalité et tend à toucher à l'ensemble de leur vie et à remettre en cause leur rapport au conflit, à l'autorité et infuse ainsi bien au-delà des limites initiales du projet. Ainsi cette fondatrice :

«Ça représente quelque chose de plus personnel, plus individuel dont beaucoup de membres de La Barbe témoignent. C'est vraiment de l'empowerment. Je ne pense pas que les gens réalisent à quel point c'est difficile de faire ça, cette simple chose de mettre une barbe, de monter sur scène, devant une assemblée, dans une hostilité. Quand tu es une femme et que tu as appris à ne pas trop hausser le ton, à être une bonne élève, à être une femme accomplie mais qui justement n'ouvre pas trop sa gueule, justement le monde ne nous a pas élevées pour

RIMHE - Revue Interdisciplinaire Management, Homme \& Entreprise $\mathrm{n}^{\circ} 26$ - Printemps 2017 


\section{La mobilisation au travail par les compétences : l'apport de l'étude des groupes activistes - Fabien HILDWEIN}

nous bagarrer. C'est très compliqué pour nous femmes d'être dans la désobéissance. Pour moi ça a aussi un sens personnel et moi je sais que chaque barbue qui monte sur scène elle le fait pour La Barbe et pour un message qui les dépasse, mais aussi ça les transforme et ça les travaille et ça leur donne aussi le courage d'ouvrir leur bouche quand il y a un machiste dans leur boulot qui dit des propos qui les défrisent, ça leur donne le courage de plein de choses, donc c'est une vraie formation à l'émancipation. Donc une action de La Barbe c'est beaucoup plus riche qu'il ne peut y paraître de l'extérieur et ça ne laisse jamais personne indemne. » [Anique - 26 février 2013]

Entrer à La Barbe, c'est se libérer d'une éducation et d'une socialisation féminines ressenties comme contraignantes. Le dévoilement concerne à la fois les situations sociales et soi-même. Ces expériences contribuent donc à l'émancipation des activistes par l'acquisition de compétences au sein de La Barbe. On n'acquiert pas ces compétences uniquement pour mieux agir au sein de l'organisation, mais aussi pour soi. L'action féministe de La Barbe n'affecte pas seulement ses cibles, mais aussi ses membres. La nature émancipatrice de ces compétences est indissociable du projet du groupe activiste, ce qui justifie l'attachement des activistes au groupe et ainsi en constitue un pilier central de mobilisation.

\subsection{Le développement mobilisateur des compétences}

Si la nature émancipatrice des compétences acquises à La Barbe explique leur caractère mobilisateur, ce n'est pas la seule raison. En effet, c'est en participant aux actions, à la communauté et par le recrutement que les activistes acquièrent et entretiennent ces compétences. Par ailleurs, les compétences elles-mêmes se renforcent mutuellement et favorisent leur acquisition.

Participer à des actions de La Barbe permet aux activistes de développer leur confiance en elles, leur aisance face à une autorité et de repenser leur rapport à l'espace.

"Quand il s'agit d'aller sur scène je dois me faire un peu violence, je dois me forcer et c'est aussi quelque chose pour lequel je remercie La Barbe parce que ça m'a obligé à sortir de moi-même et vingt fois j'ai eu le cœur battant à rompre et maintenant je ne doute absolument plus de la légitimité de notre action. » [Louise - 25 janvier 2012]

Le lien est fort entre l'action et le changement personnel : l'acte de défier une autorité et de s'imposer dans les lieux de pouvoir change les activistes. En le commettant, elles se prouvent qu'elles sont capables d'affronter cette autorité et d'encaisser l'hostilité que l'action déclenche. L'action leur permet d'explorer concrètement des capacités personnelles qu'elles n'avaient pas envisagées ou réellement expérimentées. Elles y gagnent plus de confiance en elles et développent un certain courage pour faire face aux figures d'autorité. De plus, s'imposer symboliquement et physiquement dans les lieux de pouvoir aide les activistes à le faire également dans leur vie quotidienne et à reconquérir l'espace public. Plus largement, le changement de cadre et la cause choisie 


\section{La mobilisation au travail par les compétences : l'apport de l'étude des groupes activistes - Fabien HILDWEIN}

par La Barbe sont liés : en intervenant à proximité du pouvoir et en exerçant leur esprit critique, les activistes s'autorisent à se penser comme sujets politiques.

Le deuxième mode d'acquisition des compétences se situe au niveau de la vie interne du groupe, à travers les réunions avant et après les actions, et les débats sur la liste de travail électronique. Ce mode facilite d'abord le changement de cadres et la transmission de concepts et de théories féministes, les activistes se recommandant réciproquement des lectures et en discutent. Mais il encourage également la prise de parole interne, qui contribue à développer les compétences relationnelles des activistes (débat et prise de parole en public) :

«C'est un collectif où il y a beaucoup de grandes personnalités. Il faut réussir à s'imposer, à imposer sa personnalité, face à des personnes qui savent très bien écrire, qui ont des pensées très bien construites, et quand nous on l'a un peu moins. On arrive à affirmer que là on n'est pas d'accord. J'étais incapable de le faire avant et maintenant je sens que dans le milieu professionnel, je vois l'évolution que j'ai eue, en réunion je prends beaucoup plus facilement la parole, j'affirme des choses, et je dis " c'est bizarre y'a pas de femmes », que ce soit sur ce sujet-là mais aussi sur des choses qui me perturbent et sur lesquelles je ne suis pas d'accord. » [Amelia - 20 mars 2013]

Le groupe activiste crée également un espace de parole où les activistes s'entraînent à prendre la parole. Les débats internes de La Barbe se tiennent dans des réunions nonmixtes, justement dans le but de protéger la parole des femmes qui s'y expriment. Ces débats peuvent être houleux, ce qui permet aux activistes de s'exercer d'autant mieux. Enfin, l'accueil et l'intégration des activistes sont aussi l'occasion d'acquérir des compétences. La Barbe cherche à intégrer aussi rapidement que possible les nouvelles arrivantes, en les poussant à prendre des responsabilités aussi tôt que possible : par exemple, coordonner une action ou une réunion, prendre la parole face à des journalistes au nom du groupe, etc. L'une des façons de faire consiste à établir et à maintenir un climat particulier susceptible d'inciter les activistes à acquérir de l'autonomie et de l'indépendance.

«Même si vous êtes à La Barbe depuis deux heures vous avez le droit de vous exprimer et de répondre à une interview, alors évidemment deux heures c'est un peu exagéré, mais il y a vraiment cet esprit de "on ne veut pas s'accaparer la connaissance », c'est le partage et on est un collectif et c'est l'idée d'empowerment, c'est vas-y, parce que c'est en t'y collant que tu vas aussi apprendre. » [Amelia - 20 mars 2013]

L'activiste reprend un des principes explicites du groupe : "C'est en t'y collant que tu vas apprendre », ce qui signifie que si une activiste veut quelque chose ou propose quelque chose, elle est encouragée à le réaliser elle-même. Plutôt que de reposer sur les compétences des autres, elle doit développer les siennes par la pratique, «sur le tas ». Il s'agit aussi d'un enjeu féministe: l'émancipation des femmes passe par l'acquisition de compétences permettant une plus grande autonomie sociale, notamment envers les hommes. Les activistes décrivent souvent cette prise de responsabilité comme une expérience «grisante». La Barbe agit donc comme un 


\section{La mobilisation au travail par les compétences : l'apport de l'étude des groupes activistes - Fabien HILDWEIN}

laboratoire où les activistes peuvent s'entraîner à exercer des responsabilités, même relativement modestes, au même titre que leurs débats internes leur apprennent à prendre plus facilement la parole en public.

Les compétences se renforcent réciproquement, c'est-à-dire que posséder certaines compétences facilite l'acquisition d'autres compétences. On ne peut les considérer individuellement et hors de leur contexte. Le changement de cadres est largement facilité par l'acquisition de savoirs théoriques sur le féminisme, qui apportent des explications différentes aux situations sociales. Les éléments théoriques facilitent aussi le passage à l'acte en offrant de bonnes raisons de s'y risquer, car il s'agit de briser les codes sociaux et de s'exposer dans l'action :

«Alors évidemment les gens qui nous regardent ne voient pas les réflexions théoriques. C'est très intellectuel, c'est très théorique. Quand tu es sur scène, si tu interroges le public, il y a personne qui va avoir ce degré d'analyse. Nous on sait pourquoi on le fait, on sait quel est notre parti-pris derrière, ça nous fait du bien à nous. » [Anique - 26 février 2013]

Réciproquement, les savoirs théoriques sont mis à profit par les compétences relationnelles développées durant les débats internes, au travers desquels les activistes explorent les théories et arguments féministes.

Tableau 1 : Synthèse des compétences acquises à La Barbe

\begin{tabular}{|c|c|c|c|c|}
\hline & Compétences acquises & $\begin{array}{c}\text { Type de } \\
\text { compétence }\end{array}$ & Mode d'acquisition & Facilitateurs \\
\hline \multirow{3}{*}{ 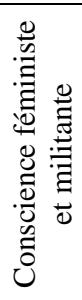 } & $\begin{array}{c}\text { Théories et concepts } \\
\text { féministes }\end{array}$ & $\begin{array}{l}\text { Savoirs } \\
\text { théoriques }\end{array}$ & $\begin{array}{l}\text { Vie interne du } \\
\text { groupe ; Lectures } \\
\text { individuelles }\end{array}$ & Débats internes \\
\hline & $\begin{array}{l}\text { Cadres d'analyse } \\
\text { féministes }\end{array}$ & $\begin{array}{l}\text { Savoir-faire } \\
\text { cognitifs }\end{array}$ & $\begin{array}{l}\text { Vie interne du } \\
\text { groupe }\end{array}$ & $\begin{array}{l}\text { Théorie et concepts } \\
\text { féministes }\end{array}$ \\
\hline & $\begin{array}{c}\text { Se penser comme sujet } \\
\text { politique et assumer } \\
\text { ses ambitions }\end{array}$ & $\begin{array}{l}\text { Savoir-faire } \\
\text { relationnel }\end{array}$ & $\begin{array}{l}\text { Participation } \\
\text { générale au groupe }\end{array}$ & $\begin{array}{l}\text { Cadres d'analyse } \\
\text { féministes }\end{array}$ \\
\hline \multirow{4}{*}{ 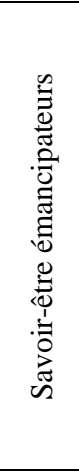 } & $\begin{array}{l}\text { Confiance en soi et } \\
\text { face à l'autorité }\end{array}$ & Savoir-être & $\begin{array}{c}\text { S'imposer } \\
\text { physiquement au } \\
\text { cours des actions }\end{array}$ & $\begin{array}{l}\text { Participation aux } \\
\text { actions }\end{array}$ \\
\hline & $\begin{array}{l}\text { Nouveau rapport à } \\
\text { l'espace public }\end{array}$ & Savoir-être & $\begin{array}{c}\text { S'imposer } \\
\text { physiquement au } \\
\text { cours des actions } \\
\end{array}$ & $\begin{array}{l}\text { Participation aux } \\
\text { actions }\end{array}$ \\
\hline & Débattre en public & $\begin{array}{l}\text { Savoir-faire } \\
\text { relationnels }\end{array}$ & $\begin{array}{l}\text { Vie interne du } \\
\text { groupe }\end{array}$ & $\begin{array}{c}\text { Espaces non-mixtes } \\
\text { protégés }\end{array}$ \\
\hline & Autonomie & Savoir-être & $\begin{array}{l}\text { Accueil des } \\
\text { nouvelles }\end{array}$ & $\begin{array}{c}\text { Développement de } \\
\text { la confiance envers } \\
\text { soi-même et envers } \\
\text { les autres }\end{array}$ \\
\hline
\end{tabular}

RIMHE - Revue Interdisciplinaire Management, Homme \& Entreprise $\mathrm{n}^{\circ} 26$ - Printemps 2017 


\section{La mobilisation au travail par les compétences : l'apport de l'étude \\ des groupes activistes - Fabien HILDWEIN}

\section{Discussion : les compétences comme levier de mobilisation}

Ainsi, les compétences peuvent être des facteurs de mobilisation lorsqu'elles s'inscrivent dans un parcours personnel, lorsque les individus les considèrent comme des ressources qu'ils peuvent mettre à leur service, à la fois pour leur développement personnel, mais également pour l'organisation elle-même. La mobilisation par les compétences fonctionne d'autant mieux que leur acquisition est enracinée dans l'activité même de l'organisation, et plus largement dans l'intégration et la socialisation des individus au sein du groupe.

Les résultats de cette étude permettent ainsi d'enrichir le lien entre fidélisation des employés et compétences. Plusieurs travaux insistent sur l'importance de conserver les compétences en fidélisant les salariés, notamment les hauts-potentiels (Dejoux et Thévenet, 2015), en particulier en temps de crise (Giraud, Roger et Thomines, 2012). Ce lien doit être complété par le constat que la fidélisation se fera aussi en formant les individus et en leur donnant la possibilité de se développer au sein de l'entreprise. Cette recherche suggère ainsi l'existence d'un cercle vertueux entre compétences et fidélisation : les individus entrent, restent et se mobilisent au sein d'une organisation pour les compétences, et en participant ainsi à l'organisation, ils renforcent leurs compétences. Pour y parvenir, la proposition d'un éventail de compétences rares, c'est-à-dire impossible à acquérir dans d'autres organisations représente un avantage concurrentiel sur le marché de l'emploi pour recruter et conserver les talents. Les compétences qui correspondent à un besoin personnel et à un parcours individuel sont particulièrement précieuses dans ce sens, en particulier autour des questions d'émancipation et de développement personnel.

Dans le cas étudié, l'engagement des activistes ne se fait pas exclusivement pour une cause, il répond aussi à un désir d'évolution personnelle intimement mêlé à leur attachement à cette cause. De la même façon, les compétences servent à la fois les individus et le groupe. Elles sont acquises conjointement par un effort individuel et par la participation à la vie générale du groupe. Plus généralement, la mobilisation par les compétences définit comment des individus s'approprient et se partagent des ressources pour rendre possible l'action collective et l'organisation existe parce qu'elle fournit à chaque individu les moyens de se mobiliser. Ainsi, les compétences relient les individus au collectif, à la fois en leur permettant d'agir pour l'organisation, mais aussi en les y attachant. Cette relation invite à dépasser les questionnements opposant les buts individuels et les buts collectifs comme dans le paradoxe d'Olson (1965) : les dynamiques comme celle de l'appropriation de ressources et l'acquisition de compétences touchent à ces deux plans et les réunissent.

La limite principale de ce travail se situe dans les différences existantes entre les groupes activistes et des organisations plus grandes et plus structurées ou, plus globalement, les entreprises. La première différence tient à la nature des individus 


\section{La mobilisation au travail par les compétences : l'apport de l'étude des groupes activistes - Fabien HILDWEIN}

étudiés. Les activistes et les employés d'une entreprise ne sont comparables que dans la mesure où il s'agit de membres d'une organisation poursuivant chacun des types de carrières spécifiques, mais ils diffèrent fondamentalement dans leur rapport à leur organisation et à leur activité. Les activistes partagent des affects (l'indignation notamment) et des cadres d'analyse qui facilitent leur coordination et leur mobilisation autour d'un projet commun, tandis que les salariés pour la plupart sont moins disposés à agir collectivement et à entretenir un rapport passionné à leur activité. De plus, les salariés sont liés par contrat et s'engagent dans une entreprise en échange d'une rémunération, tandis que les activistes sont beaucoup plus volatiles, pouvant changer d'organisation (ou même renoncer au militantisme) sans coûts pour eux ; les garder au sein de l'organisation ne dépend que de leur bon vouloir. Enfin, l'entrée d'activistes au sein d'un groupe présuppose qu'ils soient déjà mobilisés, au moins au début. L'enjeu pour le groupe activiste est donc d'abord d'entretenir la mobilisation existante et de retenir ses membres, tandis que l'enjeu pour l'entreprise est plutôt de susciter cette mobilisation avec des personnes qui s'inscrivent dans la relation d'emploi également par nécessité et non par vocation. La deuxième différence tient à la nature des compétences considérées. Les activistes tendent à valoriser des compétences qui correspondent à leur cause : l'émancipation pour le féminisme ; une expertise sur certains sujets de sciences de la nature (biologie, systémique, climatologie, physique nucléaire...) pour l'écologie ; des compétences juridiques et d'organisation pour monter une manifestation, etc. Les personnes en entreprise sont sans doute aussi touchés par cette dimension en tant que sujets politiques, voire demandeuses de la prise en compte de la défense de l'égalité professionnelle ou encore de l'écologie, mais elles restent incitées au regard des enjeux associés à l'emploi à développer des compétences transférables à d'autres postes et susceptibles de renforcer leur employabilité et leur progression de carrière.

\section{Conclusion}

L'étude de la mobilisation des activistes par les compétences ouvre la réflexion sur les bonnes pratiques de management en entreprise. Les activistes de La Barbe se sont découvertes et affirmées par leurs actions au sein de ce groupe. Elles ont acquis une forme de professionnalisme, mis en ouvre des compétences audacieuses, en ont développé de nouvelles et favorisé leur développement personnel. La transposition à l'entreprise invite à des recherches futures. Elle renvoie à l'importance du sens donné par les acteurs organisationnels à leur engagement au travail tout autant qu'à l'apprentissage permanent et à l'émancipation par le travail que représente potentiellement l'intégration dans une organisation. Elle pousse à adopter une approche des ressources détenues par les personnes comme autant de talents dont il s'agit pour le management de soutenir l'épanouissement et que peut permettre de révéler une mobilisation au travail par les compétences. 


\section{La mobilisation au travail par les compétences : l'apport de l'étude \\ des groupes activistes - Fabien HILDWEIN}

\section{Références bibliographiques}

Aït Razouk A., Bayad M. (2011), GRH mobilisatrice et performance des PME, Revue de Gestion des Ressources Humaines, vol.82, n4, p. 3-18.

Aubret J., Gilbert P., Pigeyre F. (2002), Management des compétences : réalisations concepts analyses, Paris, Dunod.

Barraud-Didier V., Guerrero S., Igalens J. (2003), L'effet des pratiques de GRH sur la performance des entreprises: le cas des pratiques de mobilisation, Revue de Gestion des Ressources Humaines, vol.47, p. 2-13.

Beaud S., Weber F. (2010), Guide de l'enquête de terrain, Paris, La Découverte.

Bertaux D. (1997), Le récit de vie, Paris, Armand Colin.

Bichon A. (2005), Comment appréhender les comportements de mobilisation collective des salariés, Gestion, vol.30, n², p. 50-59.

Bouteiller D., Gilbert P. (2005), Réflexions croisées sur la gestion des compétences en France et en Amérique du Nord, Relations industrielles/Industrial Relations, vol.60, $\mathrm{n}^{\circ} 1$, p. 3-28.

Butler J. (1990), Gender Trouble: Feminism and the Subversion of Identity, New York, Routledge.

Capaldo G., Iandoli L., Zollo G. (2006), A situationalist perspective to competency management, Human Resource Management, vol.48 n³, p. 429-448.

Chowhan J. (2016), Unpacking the black box: understanding the relationship between strategy, HRM practices, innovation and organizational performance, Human Resource Management Journal, vol.26, n², p.112-133.

Corbin J. M., Strauss A. L. (1990), Grounded theory research: Procedures, canons, and evaluative criteria, Qualitative Sociology, vol.13, $\mathrm{n}^{\circ} 1$, p. 3-21.

Cunliffe A. L. (2010), Retelling Tales of the Field, Organizational Research Methods, vol.13, n², p. 224-239.

Defélix C., Retour D. (2008), Peut-on relier stratégie d'entreprise et gestion des compétences ?, in Dupuich-Rabasse F., Management et gestion des compétences, Paris, L'Harmattan, p. 199-216.

Dejoux C., Thévenet M. (2010), La gestion des talents, La GRH d'après-crise, Paris, Dunod.

Delphy C. (1979), L'ennemi principal, 1 Economie politique du patriarcat, Paris, Editions Syllepse.

Dietrich A., Gilbert P., Pigeyre F., Aubret J. (2010), Management des compétences : enjeux, modèles et perspectives, Paris, Dunod.

Fillieule O., Péchu C. (1993), Lutter ensemble, Les théories de l'action collective, Paris, L'Harmattan.

Gilbert P. (2006), La compétence : concept nomade, significations fixes, Psychologie du Travail et des Organisations, vol.12, p. 67-77. 


\section{La mobilisation au travail par les compétences : l'apport de l'étude des groupes activistes - Fabien HILDWEIN}

Giraud L., Roger A., Thomines S. (2012), La fidélisation des ressources humaines en période de crise économique, Revue de Gestion des Ressources Humaines, vol.84, p.44-76.

Glaser B. G., Strauss A. L. (1967), The discovery of grounded theory: Strategies for qualitative research, New York, Aldine.

Goffman E. (1974), Frame Analysis, An essay on the organization of experience, Chicago, Northeastern University Press.

Green F., James D. (2003), Assessing skills and autonomy: the job holder versus the line manager, Human Resource Management Journal, vol.13, nº 1, p.63-77.

Grugulis I. (2003), Putting skills to work: learning and employment at the start of the century, Human Resource Management Journal, vol.13, n², p.3-12.

Jenkins J. C. (1985), The Policy of Insurgency, New York, Columbia University Press. Klandermans B. (2004), The Demand and Supply of Participation: SocialPsychological Correlates of Participation in Social Movements, in Snow D. A., Soule S. A., Kriesi H., Eds, The Blackwell Companion to Social Movements, Oxford, Blackwell Publishing, p. 360-379.

Koopmans R. (2004), Protest in Time and Space: The Evolution of Waves of Contention, in Snow D. A., Soule S. A., Kriesi H., The Blackwell Companion to Social Movements, Oxford, Blackwell Publishing.

Labruffe A. (2005), Management des compétences, construire votre référentiel, La Plaine Saint Denis, AFNOR éditions.

Le Boterf G. (1994), De la compétence: Essai sur un attracteur étrange, Paris, Les Editions d'Organisation.

Le Boterf G. (2002), Développer les compétences des professionnels, Paris, Editions d'Organisation.

Le Boterf G. (2010), Repenser la compétence: Pour dépasser les idées reçues : quinze propositions, Paris, Eyrolles.

Le Boterf G. (2015), Construire les compétences individuelles et collectives: Agir et réussir avec compétence, les réponses à 100 questions, Paris, Eyrolles.

Le Boulaire M., Retour D. (2008), Gestion des compétences, stratégie et performance de l'entreprise: quel est le rôle de la fonction RH ?, Revue de Gestion des Ressources Humaines, vol.70, p. 51-68.

Le Deist F.D., Winterton J. (2005), What is competence?, Human Resource Developement International, vol.8, $\mathrm{n}^{\circ} 1$, p. 27-46.

McCarthy J. D., Zald M. N. (1973), The Trend of Social Movements in America: Professionalization and Resource Mobilization, Morristown, NJ, General Learning.

McCarthy J. D., Zald M. N. (1977), Resource Mobilization and Social Movements: A Partial Theory, American Journal of Sociology, vol.82, nº ${ }^{\circ}$, p. 1212-1241. 


\section{La mobilisation au travail par les compétences : l'apport de l'étude des groupes activistes - Fabien HILDWEIN}

Michaux V. (2005), Compétences collectives et haute performance: apports théoriques et enjeux organisationnels, Revue de Gestion des Ressources Humaines, vol.58, p.45-74.

Moore S., Read I. (2006), Collective organization in small- and medium-sized enterprises - an application of mobilization theory, Human Resource Management Journal, vol.16, n 4 , p.357-375.

Oiry E., Sulzer E. (2002), Les référentiels de compétences : enjeux et formes, in Brochier D., La Gestion des Compétences. Acteurs et Pratiques, Paris, Economica, p.29-47.

Olson M. Jr. (1965), The Logic of Collective Action: Public Goods and the Theory of Groups, Cambridge, Harvard University Press.

Prahalad C.K., Hamel G. (1990), The core competence of the corporation, Harvard Business Review, vol.68, n³, p. 63-76.

Penrose E. (1963), Facteurs, conditions et mécanismes de la croissance d'une entreprise, Paris, Hommes et Techniques.

Pierru E. (2010), Organisations et Ressources, in Fillieule O., Agrikoliansky E., Sommier I., Penser les mouvements sociaux, Paris, La Découverte, p.19-38.

Retour D. (2005), Le DRH de demain face au dossier compétences, Management et Avenir, vol.2, n% 4 , p. 187-200.

Rouleau L., de Rond M., Musca G. (2014), From the ethnographic turn to new forms or organizational ethnography, Journal of Organizational Ethnography, vol.3, n ${ }^{\circ}$, p.2-9.

Sanséau P.-Y. (2005), Les récits de vie comme stratégie d'accès au réel en sciences de gestion: pertinence, positionnement et perspectives d'analyse, Recherches Qualitatives, vol.25, ${ }^{\circ} 2$, p. 33-57.

Snow D. A., Benford R. D. (1988), Ideology, Frame Resonance, and Participant Mobilization, International Social Movement Research, vol.1, p. 197-217.

Snow D. A., Rochford E. B., Worden S. K., Benford R. D. (1986), Frame Alignment Processes, Micromobilization, and Movement Participation, American Sociological Review, vol.51, n 4, p. 464-481.

Snow D. A., Soule S. A., Kriesi H. (2004), Mapping the Terrain, in Snow D. A., Soule S. A., Kriesi H., The Blackwell Companion to Social Movements, Oxford, Blackwell Publishing, p. 3-16.

Sommier I. (2010), Diffusion et circulation des mouvements sociaux, in Fillieule O., Agrikoliansky E., Sommier I., Penser les mouvements sociaux, Paris, La Découverte, p.101-120.

Suddaby R. (2009), From the editors: What grounded theory is not, Academy of Management Journal, vol.49, n ${ }^{\circ}$, p. 633-642.

Tarrow S. (1983), Struggling to reform: Social Movements and Policy Change during Cycles of Protest, Ithaca, N.Y., Western Societies Program, Cornell University.

RIMHE - Revue Interdisciplinaire Management, Homme \& Entreprise $\mathrm{n}^{\circ} 26$ - Printemps 2017 


\section{La mobilisation au travail par les compétences : l'apport de l'étude des groupes activistes - Fabien HILDWEIN}

Tarrow S. (2005), New Transnational Activism, Cambridge, Cambridge University Press.

Tarrow S., della Porta D., Dir. (2005), Transnational Protest and Global Activism, Lanham, Rowman et Littlefield.

Tremblay M., Chênevert D., Simard G., Lapalme M.-E., Doucet O. (2005), Agir sur les leviers organisationnels pour mobiliser le personnel: le rôle de la vision, du leadership, des pratiques de GRH et de l'organisation du travail, Gestion, vol.30, n 2 , p. 69-78.

Tremblay M., Simard G. (2005), La mobilisation du personnel: l'art d'établir un climat d'échanges favorable basé sur la réciprocité, Gestion, vol.30, n², p. 60-68.

Tremblay M., Wils T. (2005), La mobilisation des ressources humaines: une stratégie de rassemblement des énergies de chacun pour le bien de tous, Gestion, vol.30, n², p.37-49.

Ulrich D. (1996), Human Resource Champions. The next agenda for adding value and delivering results, Boston, Harvard Business School Press.

Watson T. J. (2011), Ethnography, Reality, and Truth: The Vital Need for Studies of "How Things Work" in Organizations and Management, Journal of Management Studies, vol.48, ${ }^{\circ} 1$, p. 202-217.

Wenerfelt B. (1984), A resource based view of the firm, Strategic Management Journal, vol 5, $\mathrm{n}^{\circ} 2$, p.171-181.

Wils T., Labelle C., Guérin C., Tremblay M. (1998), Qu'est-ce que la mobilisation des employés ? Le point de vue des professionnels en ressources humaines, Gestion, vol.23, n², p. 30-39.

\section{Annexe 1 : guide d'entretien auprès des activistes}

Première partie : Le parcours et la personnalité de l'activiste
Demander à l'activiste de se présenter : âge, occupation, origine
Avait-elle des expériences militantes préalables ?
Quelle expérience du féminisme avait-elle antérieurement ?
Comment est-elle arrivée à La Barbe et pourquoi ?
Quelle est son évolution au sein de La Barbe?
Envisage-t-elle de continuer à La Barbe ? (question rajoutée pour tenir compte des récits de départ d'autres groupes et pour mieux retracer les carrières militantes)

Deuxième partie : Le sens de la performance

Demander de décrire entièrement une performance

- Si l'explication manque, pourquoi chaque élément (postiche, banderole, ironie, etc.) ?

Quelles réactions du public?

Si possible, demander de décrire une action concrète à laquelle l'activiste et l'ethnographe ont assisté tous les deux 


\section{La mobilisation au travail par les compétences : l'apport de l'étude des groupes activistes - Fabien HILDWEIN}

\section{Annexe 2 : Liste des sources dans la presse}

Sources des articles collectés sur La Barbe entre le 28 février 2008 (création de La Barbe) et le 19 novembre 2013 (date de la recherche), soit 2077 jours, par type de journaux et par ordre alphabétique. (Entre parenthèse, le nombre d'articles parus).

- $\quad$ Presse généraliste

- 20 Minutes (5)

- $\quad$ BFM (1)

- $\quad$ Direct Matin (5)

- Huffingtonpost (3)

- $\quad$ L'Humanité (6)

- L'Humanité Dimanche (1)

- $\quad$ La Croix (3)

- $\quad$ Le Figaro (2)

- $\quad$ Le Journal du Dimanche (2)

- $\quad$ Le Monde (3)

- $\quad$ Le Nouvel Observateur (1)

- $\quad$ Le Point (4)

- $\quad$ Les Inrocks (4)

- $\quad$ Les Nouvelles News (5)

- $\quad$ L'Express (3)

- $\quad$ Libération (9)

- $\quad$ M le magazine du Monde (1)

- Marianne (1)

- $\quad$ Mediapart.fr (2)

- Metronews (1)

- $\quad$ Rue89 (3)

- Presse économique

- $\quad$ Alternatives économiques (3)

- Investir.fr (2)

- $\quad$ L'Entreprise (1)

- $\quad$ La Tribune (1)

- $\quad$ Les Echos (3)

- $\quad$ Stratégies (3)

- $\quad$ Presse locale

- $\quad$ Centre Presse (2)

- $\quad$ Dimanche Ouest France (1)

- $\quad$ La Charente Libre (7)

- $\quad$ La Nouvelle République (4)

- $\quad$ La Voix du Nord (4)

- $\quad$ L'AGEFI Quotidien (1)

- $\quad$ Le Berry Républicain (1)

- $\quad$ Le Midi Libre (3)

- $\quad$ Le Parisien (14)

- $\quad$ Le Progrès (3)

- $\quad$ L'Est Républicain (2)

- $\quad$ L'Indépendant (6)

- $\quad$ Ouest France (11)

RIMHE - Revue Interdisciplinaire Management, Homme \& Entreprise $n^{\circ} 26$ - Printemps 2017 


\section{La mobilisation au travail par les compétences : l'apport de l'étude des groupes activistes - Fabien HILDWEIN}

- $\quad$ Presse Océan (7)

- $\quad$ Sud Ouest (7)

- $\quad$ Tribune de Genève (1)

- $\quad$ Agences de presse

- $\quad$ AFP (51)

- $\quad$ Associated Press (2)

- $\quad$ ATS Information (2)

- $\quad$ Reuters (5)

- $\quad$ Presse étrangère et presse spécialisée

- $\quad$ Acteurs Publics (1)

- $\quad$ ComTex (1)

- $\quad$ Grazia (2)

- $\quad$ La Correspondance de la Presse (1)

- $\quad$ La Gazette Santé Sociale (1)

- $\quad$ La Presse Canadienne (1)

- $\quad$ Le Matin (2)

- $\quad$ Rue $69(1)$

- $\quad$ Slate (3)

- $\quad$ The Times (1) 